\title{
Correlation between platelet gelsolin levels and different types of coronary heart disease
}

\author{
LIU Yue, YIN HuiJun*, JIANG YueRong, XUE Mei \& CHEN KeJi \\ Department of Cardiovascular Disease, Xiyuan Hospital, China Academy of Chinese Medical Sciences, Beijing 100091, China
}

Received July 5, 2011; accepted November 23, 2011; published online December 16, 2011

Gelsolin is an important cytoskeletal protein of platelets and studies have shown a close relationship between gelsolin and cardiovascular disease. However, the role of gelsolin in the development of coronary heart disease (CHD) is unclear. In this study, we record the distribution of gelsolin in human platelets and plasma and its association with different types of CHD. This study included 114 cases, with 33 stable angina pectoris (SAP) cases, 81 acute coronary syndrome (ACS) cases-composed of 39 unstable angina pectoris (UAP) and 42 acute myocardial infarction (AMI) cases, and 31 healthy control participants. Gelsolin concentration in platelet rich plasma (PRP) and platelet poor plasma (PPP), actin filament (F-actin) and Gc-globulin of PPP were determined by enzyme-linked immunoadsorbent assay (ELISA). The fluorescence intensity of CD62p and cytoplasmic calcium $\left(\left[\mathrm{Ca}^{2+}\right]_{\mathrm{i}}\right)$ in human platelets measured by flow cytometry. We also used turbidimetry to detect the platelet aggregation rate (PAR). We analyzed the correlation between platelet gelsolin concentration and CD62p or plasma F-actin levels among each different patient group. Compared with the control group, the gelsolin level in PRP of UAP and AMI groups increased significantly $(P<0.01)$, while the gelsolin level in PPP of all the three patient groups decreased markedly $(P<0.01)$, and the CD62p, PAR, $\left[\mathrm{Ca}^{2+}\right]_{\mathrm{i}}$ of platelets, F-actin and Gc-globulin of the UAP and AMI groups increased significantly $(P<0.01)$. Compared with the SAP group, the gelsolin level in PRP, the PAR, $\left[\mathrm{Ca}^{2+}\right]_{i}$ of platelets and CD62p of other two groups increased significantly $(P<0.01)$, F-actin of the AMI group increased markedly $(P<0.01)$. Platelet cytoskeleton protein dynamics vary among the different types of CHD. Platelet gelsolin levels are markedly increased and accompanied by increased platelet activity, F-actin and $\left[\mathrm{Ca}^{2+}\right]_{\mathrm{i}}$ of ACS patients, while gelsolin levels in PPP are markedly lower. Abnormally increased platelet gelsolin levels show high positive correlation with the level of platelet activity. Therefore, platelet gelsolin might be a novel molecular marker and/or a new potential therapeutic target of anti-platelet therapy of ACS.

gelsolin, F-actin, coronary heart disease, platelet activation, acute coronary syndromes

Citation: $\quad$ Liu Y, Yin H J, Jiang Y R, et al. Correlation between platelet gelsolin levels and different types of coronary heart disease. Chin Sci Bull, 2012, 57: 631-638, doi: 10.1007/s11434-011-4926-9

Coronary heart disease (CHD) remains a major global public health problem. Platelets play an important role in hemostasis but are also responsible for the formation of pathogenic thrombi underlying acute clinical manifestations of vascular atherothrombotic disease. Multiple pathways, including adenosine diphosphate (ADP), thromboxane $\mathrm{A}_{2}$ $\left(\mathrm{TXA}_{2}\right)$, and thrombin are capable of activating platelets. Deregulated platelet activation can lead to the formation of platelet-rich thrombi that occlude the arterial lumen and are

*Corresponding author (email: huijunyin@yahoo.com.cn) capable of causing ischemia and cardiovascular events. Oral antiplatelet drugs are a milestone in the therapy of cardiovascular atherothrombotic diseases. The efficacy of antiplatelet drugs, such as aspirin and clopidogrel, in decreasing the risk of adverse events in CHD patients has been well studied in the past 20 years. Despite oral antiplatelet therapy, a number of adverse CHD events continue to occur. In recent years, many reports have shown a possible relationship between residual platelet activity, as measured by a variety of laboratory tests, and clinical outcomes; raising the possibility that "resistance" to oral antiplatelet drugs may under- 
lie many such adverse events [1]. These phenomena suggest that other pathways capable of stimulating platelet activation may exist. It is therefore meaningful to identify new therapeutic targets for anti-platelet therapy for CHD.

Using differential proteomics in platelets, our previous studies [2] found that gelsolin protein levels show the highest difference between the platelets of CHD patients and healthy controls. This suggests that the platelet cytoskeleton may play an important role in CHD development. Based on previous results, this paper further studied the distribution of gelsolin in human platelets and plasma, and studied any potential correlation with different types of CHD to verify the role of platelet gelsolin in CHD development.

\section{Methods}

\subsection{Diagnostic criteria}

Patient diagnosis meet the standards established under the ACC/AHA/ACP-ASIM Guidelines (1999) [3] for the management of patients with chronic stable angina, and the ACC/AHA Guidelines (2002) [4] for the management of patients with unstable angina and non-ST-segment elevation myocardial infarction. We selected those patients having symptoms of angina and/or objective evidence of myocardial ischemia and those with at least one marked coronary stenosis $(>50 \%)$ shown by coronary arteriography examination.

\subsection{Inclusion and exclusion criteria}

The inclusion standard required that patients must meet all the following criteria, namely, (1) falls under one of the various classifications of ischemic heart diseases; (2) suffering from angina pectoris symptoms and/or showing signs of myocardial ischemia; (3) latest coronary arteriography examination showing significant stenosis ( $>50 \%)$; and, (4) age between 35 to 75 years.

Patients who met any one of the following conditions were excluded: severe valvuloplasty, diabetes mellitus type I, presence of severe primary diseases of the liver, kidney, hemopoietic system, presence of malignant tumors, calcium channel blockers (CCB) administration in the last two weeks, inability to obtain informed consent or deemed poor compliance case, participation in other clinical trials, and pregnant or lactating women.

Subjects enrolled in the healthy control group (31 cases) were identified as healthy by history and physical examination. Tests included routine blood count, liver function, chest X-ray, and electrocardiograph (ECG), no history of prior administration of drugs affecting atherosclerotic or thrombotic processes in the last two weeks, no history of significant mental or physical diseases nor history of familiar or self-psychiatric illnesses. This study is in accordance with the Helsinki Declaration [5], with no contravention to medical ethics.

\subsection{Blood preparation}

Fresh blood (12 mL) was drawn from an antecubital vein and collected into vacutainer tubes containing acid-citratedextrose (ACD) $9 \% \mathrm{v} / \mathrm{v}$ (trisodium citrate $22.0 \mathrm{~g} / \mathrm{L}$, citric acid $8.0 \mathrm{~g} / \mathrm{L}$, dextrose $24.5 \mathrm{~g} / \mathrm{L}$ ) as anticoagulant, and the initial $2 \mathrm{~mL}$ of blood discarded to avoid spontaneous platelet activation. We collected the blood of patients before antithrombotic therapy and angiography examination. The blood was centrifuged for $10 \mathrm{~min}$ at $150 \times g$ at room temperature to obtain platelet-rich plasma (PRP) and the remaining blood centrifuged for $20 \mathrm{~min}$ at $800 \times g$ to obtain platelet poor plasma (PPP).

\subsection{Determination of CD62p expression in platelets}

We determined the level of platelet activation, as represented by the fluorescent intensity of platelet glycoprotein CD62p, by flow cytometry (EPICS Elite, Beckman Coulter Inc., Fullerton, CA, USA). We mixed the blood evenly and completed the following procedures within $4 \mathrm{~h}$. Control and test tubes containing $50 \mu \mathrm{L}$ of blood had $20 \mu \mathrm{L}$ of homotype control CD61-FITC/CD62p-PE (CD61-FITC, Anti-GPIIIa, Cat: 348093; CD62p-PE, Anti-GMP-140, Cat: 348107; Becton Dickinson Co., Franklin Lakes, NJ, USA) added. Samples were mixed evenly by light shaking, and incubated in darkness at room temperature for $20 \mathrm{~min}$. One milliliter of cold $\left(2-8^{\circ} \mathrm{C}\right)$ fixative liquid was added into each tube, mixed well and incubated again in darkness at $2-8^{\circ} \mathrm{C}$ for $30 \mathrm{~min}$. Samples were then analyzed by flow cytometry to measure the mean fluorescence intensity (MFI) of CD62p. We used multi-parameter and multi-fluorescent flow cytometry with standard fluorescent micro-balloon adopted to correct the beam path and stream. Test data was obtained by Forward Scatter (FSC) vs. Side Scattering (SSC) gate, flow cytometry special software (Expo32, Beckman Coulter Inc.).

\subsection{Determination of platelet aggregation rate (PAR)}

We determined the PAR among different patients groups using turbidimetry (Platelet Aggregation Instrument, LBYNJ2, Beijing Lipusheng Co., China). The inducer of platelet aggregation is arachidonic acid (AA, Helena Biosciences Co., Sunderland, Tyne and Wear, UK).

\subsection{Determination of $\left[\mathrm{Ca}^{2+}\right]_{i}$ in platelets}

Platelet-rich plasma was prepared and incubated with $4 \mu \mathrm{mol} / \mathrm{L}$ Fluo-3-AM (Sigma, Saint Louis, MO, USA) at $37^{\circ} \mathrm{C}$ for $40 \mathrm{~min}$. The $\mathrm{Ca}^{2+}$ concentration of platelets was determined using flow cytometry to measure their MFI, as previously described [6]. The platelet $\mathrm{Ca}^{2+}$ concentration $(\mathrm{nmol} / \mathrm{L})$ is calculated as $\left[\mathrm{Ca}^{2+}\right]_{\mathrm{i}}=K_{\mathrm{d}} \times\left(F-F_{\min }\right) /\left(F_{\max }-F\right)[7]$. 


\subsection{Determination of gelsolin, F-actin and Gc-globulin}

The plasma concentration (PRP and PPP) of gelsolin (Cat: E0372h, R\&D Co., Minneapolis, MN, USA), F-actin (Cat: E1876h, R\&D Co.) and Gc-globulin (Cat: E1910h, R\&D Co.) were determined by enzyme-linked immunoadsorbent assay (ELISA), as per the manufacturer's instructions.

\subsection{Statistical analysis}

Statistical analysis was conducted using SPSS 13.0 software package. Data are expressed as mean \pm standard deviation $(\bar{x} \pm s)$ or counts (percentage), unless otherwise specified. We used Chi-square or rank sum test to evaluate enumeration data. For measurement data, we used variance analysis or $t$-tests. We assessed correlations of platelet gelsolin with other variables by Spearman correlation coefficient and unitary linear regression. A $P$-value of $<0.05$ was considered statistically significant.

\section{Results}

\subsection{General clinical materials}

All CHD patients enrolled were in-patients admitted from August 2010 to February 2011 in Beijing Anzhen Hospital of Capital Medical University and were diagnosed and assigned to the SAP (33 cases), UAP (39 cases) and AMI (42 cases) groups accordingly.

There was no statistical difference among the three groups in age, sex and platelet count $(P>0.05)$. The risk factors (smoking, hypertension, dyslipidemia and diabetes history), location of affected coronary artery and medication history (statins and aspirin) was comparable among the three groups $(P>0.05)$ (Table 1).

\subsection{Comparison of plasma gelsolin concentration of CHD patients among each patient group}

Compared with the control group, the gelsolin concentration in the PRP of the UAP and AMI groups was significantly higher $(P<0.01)$, while that in the PPP of the three patients groups decreased markedly $(P<0.01)$. Compared with the SAP group, the gelsolin concentration in the PRP of the UAP and AMI groups was significantly higher $(P<0.01)$ (Table 2).

\subsection{Comparison of CD62p and $\left[\mathrm{Ca}^{2+}\right]_{i}$ of platelets for CHD patients among each patient group}

Compared with healthy controls, the CD62p and $\left[\mathrm{Ca}^{2+}\right]_{\mathrm{i}}$ of platelets was significantly higher $(P<0.01)$ among the three

Table 1 Clinical features of each study groups

\begin{tabular}{|c|c|c|c|c|}
\hline \multirow{2}{*}{ Parameter } & \multicolumn{3}{|c|}{ CHD patients } & \multirow{2}{*}{$\begin{array}{l}\text { Control group } \\
\quad \text { (31 cases) }\end{array}$} \\
\hline & SAP group (33 cases) & UAP group (39 cases) & AMI group (42 cases) & \\
\hline Age (years, $\bar{x} \pm s$ ) & $54.0 \pm 6.5$ & $52.5 \pm 5.5$ & $51.5 \pm 7.5$ & $50.5 \pm 8.0$ \\
\hline Male/females (case) & $26 / 7$ & $30 / 9$ & $35 / 7$ & $23 / 8$ \\
\hline \multicolumn{5}{|l|}{ Risk factor [case (\%)] } \\
\hline Hypertension & $15(45.5)$ & $17(43.6)$ & $20(47.6)$ & 0 \\
\hline Diabetes & $4(12.1)$ & $4(10.3)$ & $5(11.9)$ & 0 \\
\hline Current smokers & $22(66.7)$ & $25(64.1)$ & $29(69.05)$ & 0 \\
\hline \multicolumn{5}{|l|}{ Medication [case (\%)] } \\
\hline Aspirin & $20(60.6)$ & $24(61.5)$ & $26(61.9)$ & 0 \\
\hline Statins & $13(39.4)$ & $15(38.5)$ & $17(40.5)$ & 0 \\
\hline Single-vessel disease [case $(\%)]$ & $12(36.4)$ & $15(38.5)$ & $16(38.1)$ & 0 \\
\hline Platelet count $\left(\times 10^{3} / \mu \mathrm{L}\right)$ & $214 \pm 56.9$ & $215 \pm 38.8$ & $216 \pm 22.8$ & $210.9 \pm 42.7$ \\
\hline
\end{tabular}

Table 2 Comparison of plasma gelsolin concentration among different patient groups $(\bar{x} \pm s, \mu \mathrm{g} / \mathrm{mL})^{\mathrm{a})}$

\begin{tabular}{cccc}
\hline Group & Case & PRP & PPP \\
SAP & 33 & $105.13 \pm 15.27^{* *}$ & $109.17 \pm 18.12^{* *}$ \\
UAP & 39 & $128.23 \pm 17.55^{* *+\dagger}$ & $112.58 \pm 11.47^{* *}$ \\
AMI & 42 & $162.16 \pm 21.07^{* *+\dagger}$ & $112.40 \pm 8.66^{* *}$ \\
Control & 31 & $117.55 \pm 9.91$ & $121.44 \pm 6.77$ \\
\hline
\end{tabular}

a) **, $P<0.01$, compared with the control group; $\dagger \dagger, P<0.01$, compared with the SAP group. 
patient groups. Compared with the SAP group, the CD62p and $\left[\mathrm{Ca}^{2+}\right]_{i}$ of platelets among the UAP and AMI groups was significantly higher $(P<0.01)$ again (Figures 1 and 2$)$.

\subsection{Comparison of PAR for CHD patients among each patient group}

Compared with healthy controls, the PAR of the three patient groups was markedly increased $(P<0.01)$. Compared with the SAP group, the PAR of the UAP and AMI groups was significantly increased $(P<0.01)$ again (Figure 3 ).
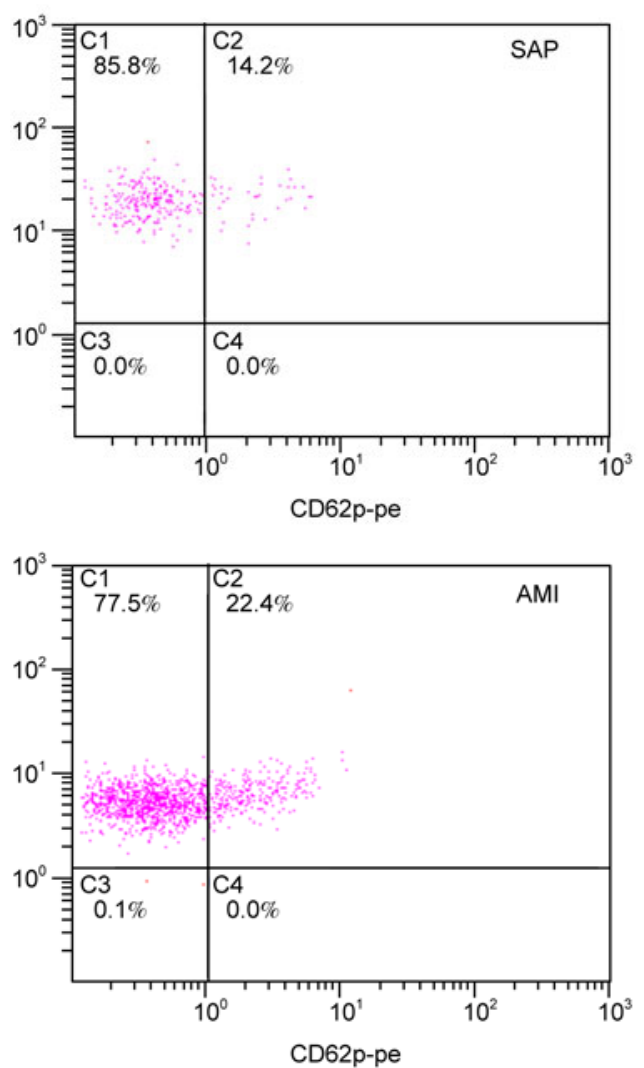

\subsection{Comparison of F-actin and Gc-globulin concentra- tion for CHD patients among each patient group}

Compared with healthy controls, the F-actin of the UAP and AMI groups was significantly increased $(P<0.05$ and $P<$ 0.01 respectively). The Gc-globulin of the three CHD groups was also significantly higher $(P<0.01)$. Compared with the SAP group, the F-actin of the AMI group was significantly increased $(P<0.01)$. However, the Gc-globulin of the other two groups was not statistically different $(P>0.05)$ (Figure 4).
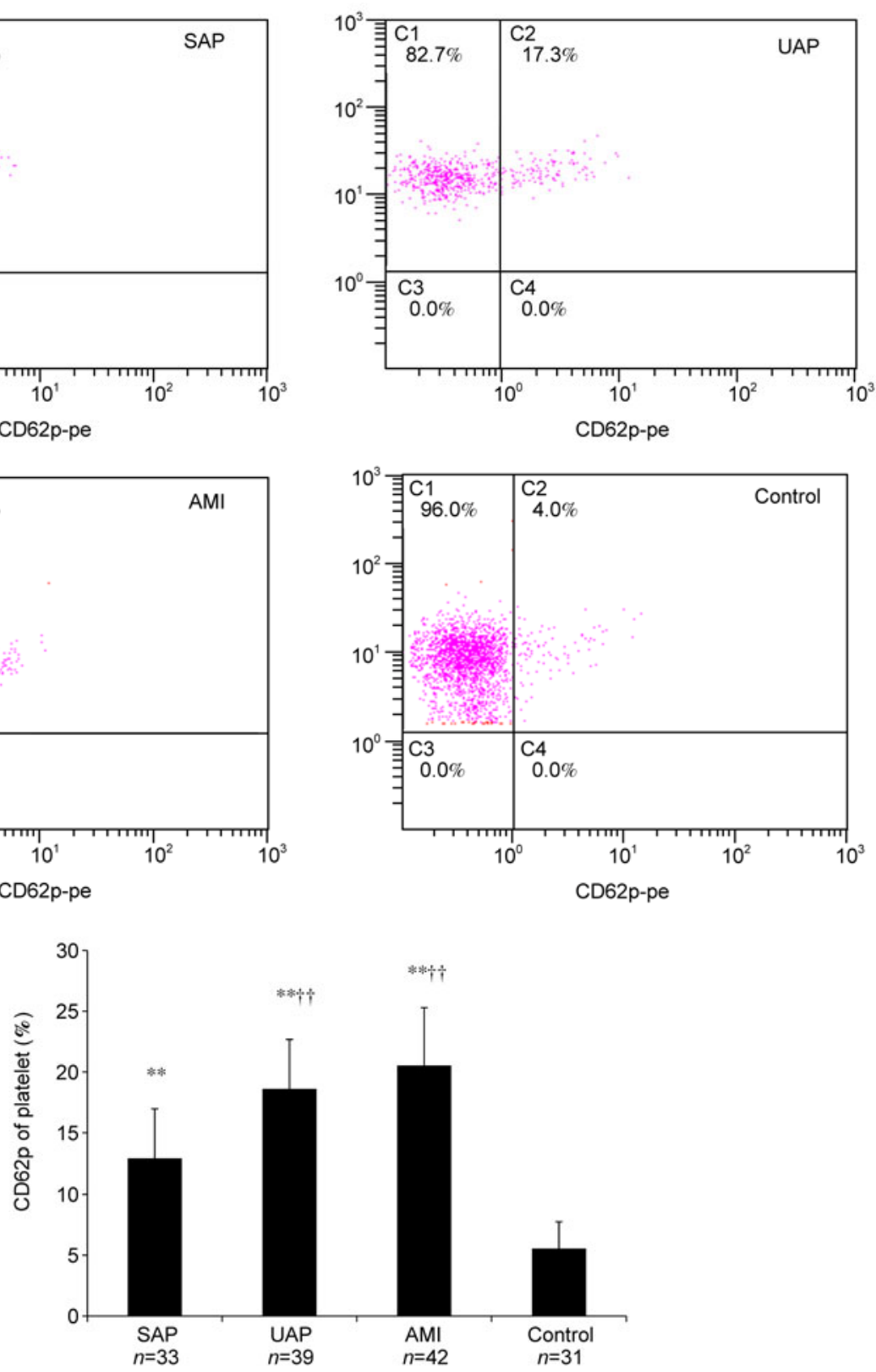

Figure 1 Comparison of CD62p of platelet for CHD patients among different groups (mean \pm standard deviation). SAP group, $12.97 \% \pm 4.01 \%$; UAP group, $18.62 \% \pm 4.05 \%$; AMI group, $20.54 \% \pm 6.75 \%$; Control group, $5.55 \% \pm 2.20 \%$. **, $P<0.01$, compared with the control group; $\dagger \dagger, P<0.01$, compared with the SAP group. 

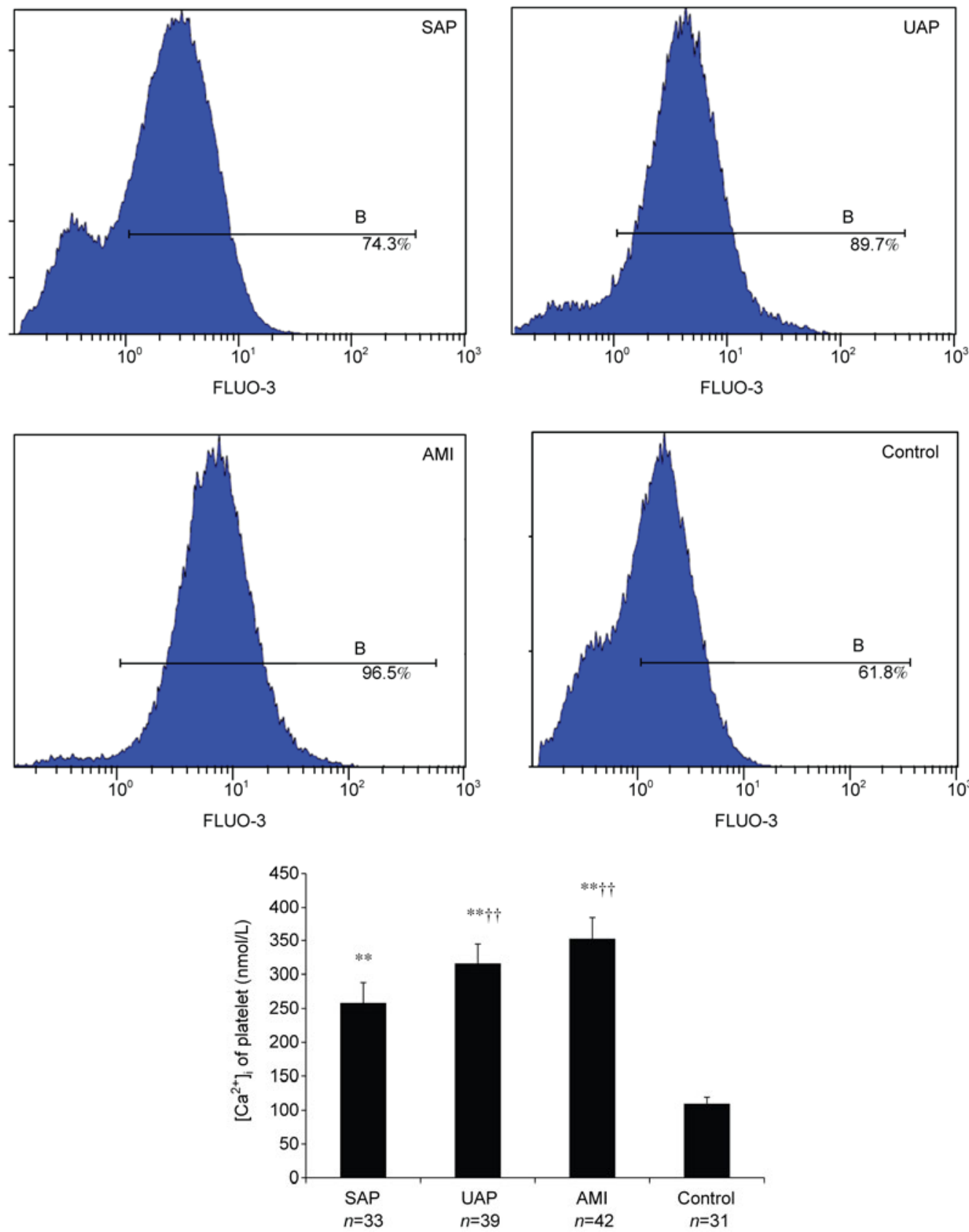

Figure 2 Comparison of $\left[\mathrm{Ca}^{2+}\right]_{\mathrm{i}}$ of platelet for CHD patients among different groups (mean \pm standard deviation). SAP group, $258.22 \pm 30.19 \mathrm{nmol} / \mathrm{L} ; \mathrm{UAP}$ group, $315.98 \pm 28.88 \mathrm{nmol} / \mathrm{L}$; AMI group, $352.51 \pm 32.51 \mathrm{nmol} / \mathrm{L}$; Control group, $109.62 \pm 9.84 \mathrm{nmol} / \mathrm{L}$. **, $P<0.01$, compared with the control group; $\dagger \dagger, P<$ 0.01 , compared with the SAP group.

\subsection{Analyses of correlations between platelet gelsolin concentration and CD62p or plasma F-actin levels among each patient group}

Next, we investigated any potential correlation between the platelet gelsolin concentration and CD62p or plasma F-actin levels that may exist between the SAP, UAP and AMI groups. Correlation analysis showed that platelet gelsolin concentrations were high positively correlated with CD62p or plasma F-actin levels in each of the three patient groups (Figure 5).

\section{Discussion}

CHD is a leading cause of death in many developed countries. It is increasingly clear that cardiovascular outcomes depend on an understanding of the biology of CHD, which may involve vascular inflammation, endothelial dysfunction, and plaque instability [8], among others. Activated platelets play a pivotal role in the formation of arterial thrombi [9]. CD62p (P-selection) is a $140 \mathrm{kD}$ glycoprotein that is present in the granules of platelets and translocates rapidly to the 


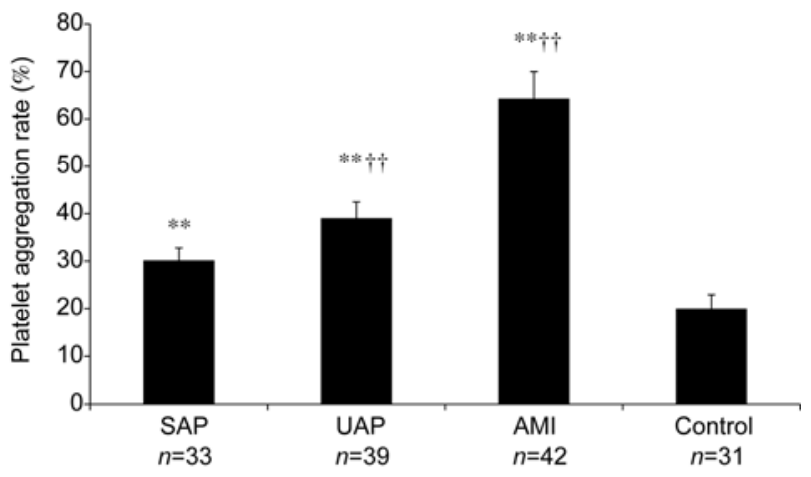

Figure 3 Comparison of platelet aggregation rate (PAR) for CHD patients among different groups (mean \pm standard deviation). SAP group, $30.18 \% \pm 2.6 \%$; UAP group, $39.07 \% \pm 3.41 \%$; AMI group, $64.29 \% \pm 5.67 \%$; Control group, $20 \% \pm 2.93 \%$. **, $P<0.01$, compared with the control group; $\dagger \dagger, P<0.01$, compared with the SAP group.
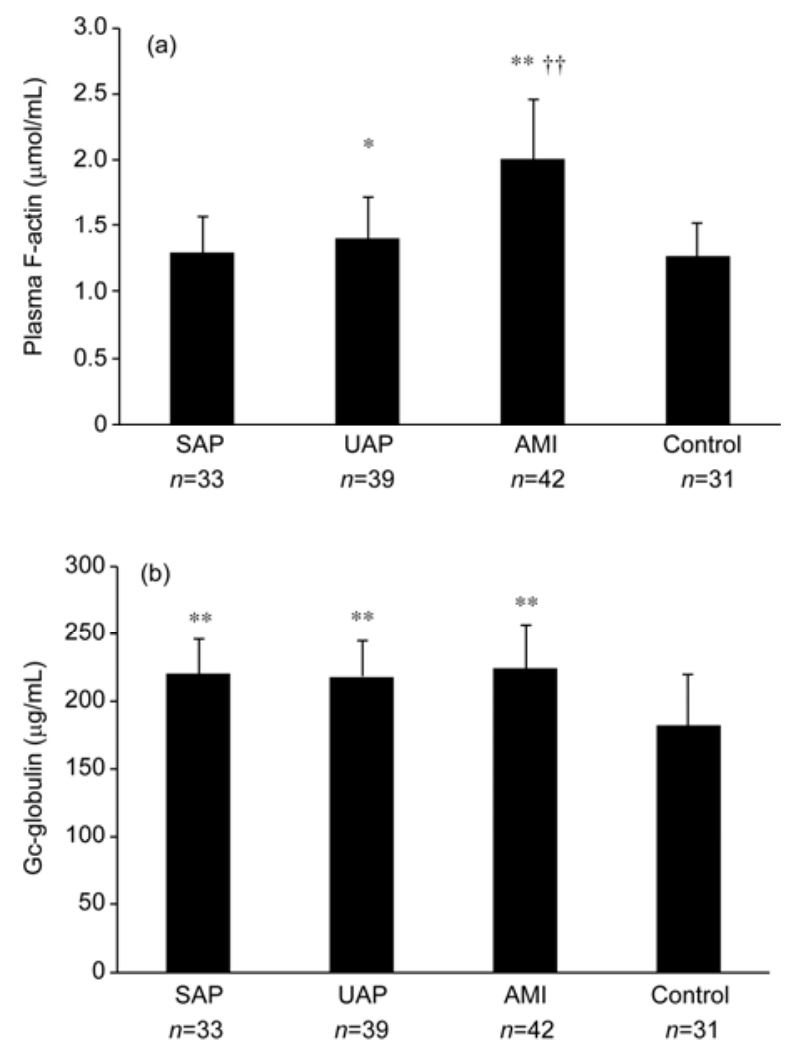

Figure 4 Comparison of F-actin and Gc-globulin concentration for CHD patients among different groups (mean \pm standard deviation). (a) Plasma F-actin concentration for CHD patients among different groups. SAP group, $1.29 \pm 0.29 \mu \mathrm{mol} / \mathrm{mL}$; UAP group, $1.41 \pm 0.31 \mu \mathrm{mol} / \mathrm{mL}$; AMI group, $2.01 \pm$ $0.46 \mu \mathrm{mol} / \mathrm{mL}$; CONTROL group, $1.27 \pm 0.25 \mu \mathrm{mol} / \mathrm{mL}$. (b) Plasma Gcglobulin concentration for CHD patients among different groups. SAP group, $220 \pm 26.78 \mu \mathrm{g} / \mathrm{mL}$; UAP group, $218.27 \pm 27.30 \mu \mathrm{g} / \mathrm{mL}$; AMI group, $224.49 \pm 32.73 \mu \mathrm{g} / \mathrm{mL}$; CONTROL group, $181.81 \pm 37.67 \mu \mathrm{g} / \mathrm{mL}$. *, $P<0.05$, **, $P<0.01$, compared with control group; $\dagger \dagger, P<0.01$, compared with SAP group.

cell surface after platelet activation, and is generally considered to be the gold marker of platelet activation $[10,11]$. There is evidence that patients with various types of CHD, including stable [12] and unstable [13] angina and acute myocardial infarction [14] have increased CD62p levels. Among all types of CHD, symptomatic stable angina is a clinical expression of myocardial ischemia associated with fixed atherosclerotic coronary stenosis; however, patients with acute coronary syndrome (ACS) constitute the major proportion of persons who require admission to cardiac units for urgent care, including invasive treatment and aggregate anticoagulant and antiplaque drug therapy [15]. In this study, our results were consistent with these observations, indicating that varying degrees of platelet activation appear in all types of CHD and that ACS (including unstable angina and non-ST elevation MI) has a higher level of platelet activation and PAR compared with that of stable angina. These findings suggest an important role for CD62p in the arterial thrombosis of ACS.

Platelet activation not only causes membrane protein change, but also a series of morphological changes, from inviscid, discotic circulating platelets into a paste-like, protruding platelet jelly, that depends on the regulation of platelet cytoskeletal proteins.

Over the past ten years, laboratories have successfully applied proteomics technology to platelet research, contributing to the emerging field of platelet proteomics. Those studies led to the identification of a considerable number of novel platelet proteins, many of which have been studied further at a functional level [16]. Our previous study [2] had identified gelsolin as having the greatest difference in expression levels between the platelets of CHD patients and healthy subjects. However, the role of platelet gelsolin in the development of CHD is unclear.

Gelsolin is a calcium-activated F-actin severing and capping protein found in many cell types and that is expressed as both cytoplasmic and plasma isoforms, and is an important cytoskeletal protein [17]. Two regulatory mechanisms are thought to modulate gelsolin activity in vivo. Calcium activates gelsolin to allow capping and severing of F-actin, while phosphatidylinositol-4,5-bisphosphate $\left(\mathrm{PIP}_{2}\right)$ at the cell membrane keeps gelsolin sequestered in an inactive state. Upon hydrolysis of $\mathrm{PIP}_{2}$, gelsolin is released into the cytoplasm and $\mathrm{Ca}^{2+}$ dependent activation can occur [18]. Previous work on gelsolin has mainly focused on the calcium regulated remodeling of F-actin, while more recent data has begun to elucidate the importance of gelsolin-mediated function in pathological conditions, including cardiovascular disease [19].

During tissue injury and cell death, actin is released into the circulation where it can interact with components of the haemostatic and fibrinolytic systems, or polymerize and form F-actin. In vitro studies [20] have suggested that F-actin can lead to platelet aggregation directly, and the presence of F-actin in blood vessels, which can plug smaller vessels and decrease blood flow to promote the formation of blood clots, can be fatal. Infusion of high doses of G-actin in rabbits caused the rapid and fatal formation of massive actin filament-containing thrombi in arterioles and capillaries of 
(a)

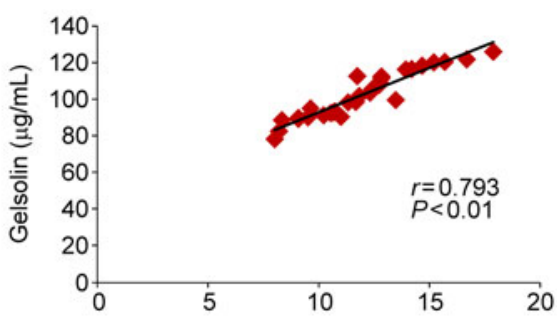

(b)

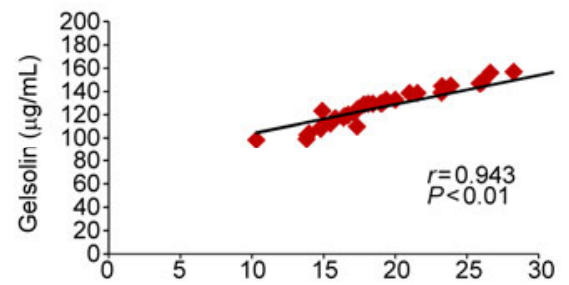

(c)

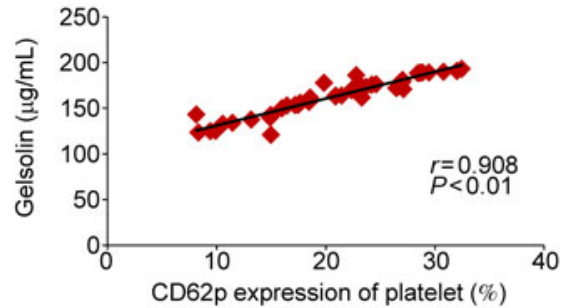

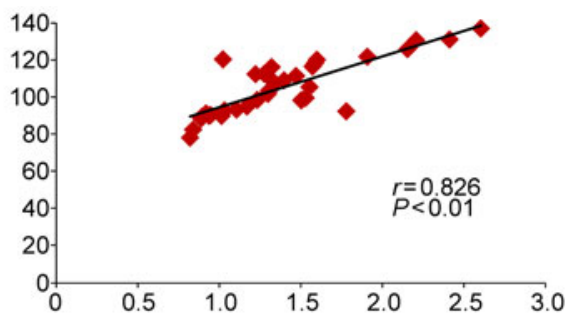
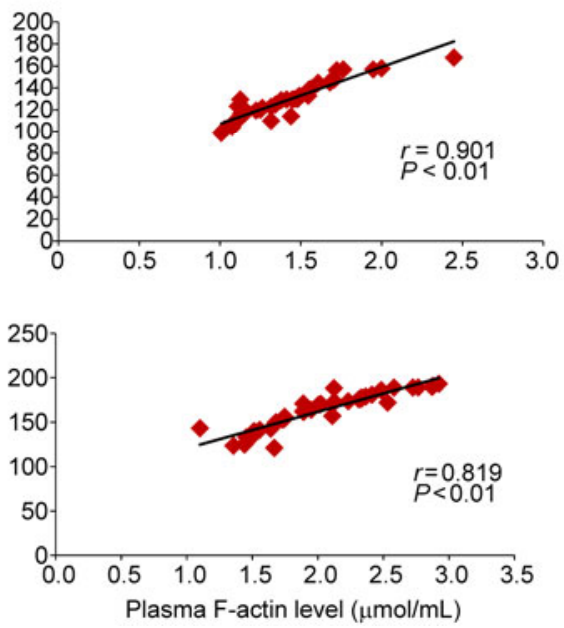

Figure 5 Analysis of correlations between platelet gelsolin concentration and CD62p or plasma F-actin level among different groups. (a) SAP group $(n=33)$; (b) UAP group $(n=39)$; (c) AMI group $(n=42)$.

pulmonary veins, as well as endothelial injury [21]. An actin scavenger system [22] is therefore likely to exist, plasma gelsolin, together with Gc-globulin, another extracellular actin-binding protein, were regarded as potentially important components of this system: capable of removing F-actin from the circulation and inhibiting F-actin elongation.

Our results were consistent with previous studies and showed that the gelsolin concentration of PPP decreased in SAP and ACS patients [23]. ACS is often associated with the rupture of vulnerable atherosclerotic plaques and coronary thrombus formation, leading to adverse outcomes. The results of this study clearly demonstrated that actin is released into the bloodstream, leading to accumulation of F-actin at the higher level of platelet activation of ACS, and that circulating actin concentrations in excess of plasma gelsolin or Gc-globulin have prothrombotic or cytotoxic activities resulting in the severe depletion of plasma gelsolin. Gc-globulin (vitamin D binding protein) is a multifunctional protein [24]; its main physiological importance is probably the binding of actin, or binding and transportation of vitamin D analogous. Interestingly, in this study we observed that the plasma of CHD patients contains greater amounts of Gc-globulin compared with healthy subjects. Previous studies [25] demonstrated that three vitamin D binding protein isotypes were increased in asprin-resistant CHD patients and this could reduce the inhibitory effect of asprin on thromboxane $\mathrm{A}_{2}$ production. Thus, this may suggest that the increased Gc-globulin level in this study may be associated with a large number of asprin-resistant patients included in our study or with increased reactively due to sudden severe depletion.

Another finding from our present study is the different levels of gelsolin in human platelet and plasma. Namely, an increase in platelet gelsolin levels, which was in agreement with our findings from a study of platelet proteomics [2], and a decrease in plasma gelsolin levels in ACS patients, compared to healthy subjects and stable angina patients. An analysis between platelet gelsolin concentrations and CD62p showed that platelet gelsolin levels have a high positive correlation with the level of platelet activation in ACS. Calcium ions not only promote gelsolin secretion but also play a vital role in the development of platelet activation. Studies have shown increased platelet $\left[\mathrm{Ca}^{2+}\right]_{\mathrm{i}}$ in patients with CHD [26], and that calcium chelators can reduce platelet $\left[\mathrm{Ca}^{2+}\right]_{\mathrm{i}}$ and inhibit platelet aggregation [27]. Our results showed an increased platelet $\left[\mathrm{Ca}^{2+}\right]_{\mathrm{i}}$ in ACS compared with healthy subjects and stable angina patients. The increased calcium influx in platelets could be one of the main mechanisms causing the abnormally increased platelet gelsolin levels observed in ACS.

Previous research has focused on gelsolin's possible role in cardiovascular diseases; several directions are currently being explored to test whether gelsolin itself can be used as a therapeutic molecule [28]. Our findings provide further insight into the important role of platelet gelsolin in the process of platelet activation in ACS. In this study, we have 
shown that platelet cytoskeletal proteins vary between different types of CHD, manifested mainly in gelsolin and F-actin. It is likely that plasma gelsolin clears away a great deal of F-actin released into circulation, and this is consistent with a role as an actin scavenger. At the onset of ACS, plasma gelsolin levels diminish significantly, while platelet gelsolin level increased abnormally, accompanied by an increased level of platelet activation and calcium influx. Platelet gelsolin levels are more highly correlated with ACS, compared to SAP. Therefore, does platelet gelsolin represent a novel therapeutic target of anti-platelet activation in ACS? We believe this is a significant area of further study, and it will be our research direction in the future.

Acknowledgment is due to Dr. LIU Hong (Director, Department of Internal Medicine) and Dr. LIU Xin (Cardiology Consulting Physician) of Beijing Anzhen Hospital for their assistance during the clinical case recruitment process. Also to XU Yong-Gang (Assistant Research Fellow) of Hematology Laboratory of Xiyuan Hospital for his assistance in the detection of CD62p expression and $\left[\mathrm{Ca}^{2+}\right]_{i}$ in platelets. This work was supported by the National Natural Science Foundation of China (81073086 and 30901949).

1 Gergely F, Andrea F, Gabriella P, et al. Clinical importance of aspirin and clopidogrel resistance. World J Cardiol, 2010, 2: 171-186

2 Li X F, Jiang Y R, Wu C F, et al. Study on the correlation between platelet function proteins and symptom complex in coronary heart disease (in Chinese). Mol Cardiol China, 2009, 9: 326-331

3 Gibbons R J, Chatterjee K, Daley J, et al. ACC/AHA/ACP-ASIM guidelines for the management of patients with chronic stable angina: A report of the American college of cardiology/American Heart Association task force on practice guidelines (committee on management of patients with chronic stable angina). J Am Coll Cardio, 1999, 33: 2092-2197

4 Braunwald E, Antman E M, Kupersmith J W, et al. ACC/AHA 2002 guideline update for the management of patients with unstable angina and non-ST-segment elevation myocaridal infarction-summary article: A report of the American college of cardiology/American Heart Association task force on practice guidelines (committee on the management of patients with unstable angina). J Am Coll Cardio, 2002, 40: $1266-1374$

5 World Medical Association declaration of Helsinki. Recommendations guiding physicians in biomedical research involving human subjects. JAMA, 1997, 277: 925-926

6 Zhuang M M, Wen Y X, Liu S L, et al. Determination of the level of cytopplasmic free calcium in human platelets with flow cytometry (in Chinese). J Xi' an Jiaotong Univ (Med Sci), 2005, 26: 508-510

7 Grynkiewicz G, Poenie M, Tsien R Y. A new generation of $\mathrm{Ca}^{2+}$ indicators with greatly improved fluorescence properties. J Biol Chem, 1985, 260: 3440-3450

8 Libby P. Inflammation and cardiovascular disease mechanisms. Am J Clin Nutr, 2006, 83: 456S-460S

9 Brydon L, Magid K, Steptoe A. Platelets, coronary heart disease, and stress. Brain Behav Immun, 2006, 20: 113-119
10 Hsu-Lin S, Berman C L, Furie B C, et al. A platelet membrane protein expressed during platelet activation and secretion: Studies using a monoclonal antibody specific for thrombin-activated platelets. J Biol Chem, 1984, 259: 9121-9126

11 Michelson A D, Furman M I. Laboratory markers of platelet activation and their clinical significance. Curr Opin Hematol, 1999, 6: 342-348

12 Furman M I, Benoit S E, Barnard M R, et al. Increased platelet reactivity and circulating monocyte-platelet aggregates in patients with stable coronary artery disease. J Am Coll Cardiol, 1998, 31: 352-358

13 Ikeda $\mathrm{H}$, Takajo $\mathrm{Y}$, Ichiki $\mathrm{K}$, et al. Increased soluble form of P-selectin in patients with unstable angina. Circulation, 1995, 92: 1693-1696

14 Shimomura H, Ogawa $\mathrm{H}$, Arai $\mathrm{H}$, et al. Serial changes in plasma levels of soluble P-selectin in patients with acute myocardial infarction. Am J Cardiol, 1998, 81: 397-400

15 Lichtman J H, Bigger J T Jr, Blumenthal J A, et al. Depression and coronary heart disease. Recommendations for screening, referral, and treatment: A science advisory from the American Heart Association Prevention Committee of the Council on Cardiovascular Nursing, Council on Clinical Cardiology, Council on Epidemiology and Prevention, and Interdisciplinary Council on Quality of Care and Outcomes Research. Endorsed by the American Psychiatric Association. Circulation, 2008, 118: 1768-1775

16 García A. Clinical proteomics in platelet research: Challenges ahead. J Thromb Haemost, 2010, 8: 1784-1785

17 Kwiatkowski D J, Stossel T P, Orkin S H, et al. Plasma and cytoplasmic gelsolins are encoded by a single gene and contain a duplicated actin-binding domain. Nature, 1986, 323: 455-458

18 Allen, P G. Actin filament uncapping localizes to ruffling lamellae and rocketing vesicles. Nat Cell Biol, 2003, 5: 972-979

19 Liu Y, Jiang Y R, Yin H J, et al. Gelsolin and cardiovascular diseases (in Chinese). Mol Cardiol China, 2011, 11: 50-53

20 Vasconcellos C A, Lind S E. Coordinated inhibition of actin-induced platelet aggregation by plasma gelsolin and vitamin D-binding protein. Blood, 1993, 82: 3648-3657

21 Haddad J G, Harper K D, Guoth M, et al. Angiopathic consequences of saturating the plasma scavenger system for actin. Proc Natl Acad Sci USA, 1990, 87: 1381-1385

22 Lee W M, Galbraith R M. The extracellular actin-scavenger system and actin toxicity. N Engl J Med, 1992, 326: 1335-1341

23 Suhler E, Lin W, Yin H L. Decreased plasma gelsolin concentrations in acute liver failure, myocardial infarction, septic shock and myonecrosis. Crit Care Med, 1997, 25: 594-598

24 White $\mathrm{P}$, Cooke $\mathrm{N}$. The multifunctional properties and characteristics of vitamin D-binding protein. Trends Endocrinol Metabol, 2000, 11: 320-327

25 López-Farré A J, Mateos-Cáceres P J, Sacristán D, et al. Relationship between vitamin $\mathrm{D}$ binding protein and aspirin resistance in coronary ischemic patients: A proteomic study. J Proteome Res, 2007, 6: 2481-2487

26 Kato M, Kambe M, Kajiyama G. Increased cytosolic free $\mathrm{Mg}^{2+}$ and $\mathrm{Ca}^{2+}$ in platelets of patients with vasospastic. Am J Physiol, 1998, 274: 548-554

27 Fujinishi A, Takahara K, Ohba C, et al. Effects of nisoldipine on cytosolic calcium, platelet aggregation, and coagulation/fibrinolysis in patients with coronary artery disease. Angiology, 1997, 48: 515-521

28 Li G H, Shi Y, Chen Y, et al. Gelsolin regulates cardiac remodeling after myocardial infarction through DNase I-mediated apoptosis. Circ Res, 2009, 104: 896-904

Open Access This article is distributed under the terms of the Creative Commons Attribution License which permits any use, distribution, and reproduction in any medium, provided the original author(s) and source are credited. 